УДК 622.244.442.063

АНАЛИЗ СПОСОБОВ СНИЖЕНИЯ ВЯЗКОСТИ НЕНЬЮТОНОВСКОЙ НЕФТИ НА ОСНОВЕ ФРАКТАЛЬНОЙ ТЕОРИИ

\title{
ANALYSIS OF WAYS TO REDUCE NON-NEWTONIAN OIL VISCOSITY BASED ON FRACTAL THEORY
}

\section{В.И. Лесин}

Институт проблем нефти и газа Российской Академии наук, г. Москва, Российская Федерация

$$
\text { С.В. Лесин }
$$

Российский государственный университет нефти и газа им. И.М. Губкина, г. Москва, Российская Федерация

\section{Viktor I. Lesin}

Oil and Gas Research Institute, Russian Academy of Sciences, Moscow, Russian Federation

$$
\text { Sergey V. Lesin }
$$

National University of Oil and Gas «Gubkin University»,

$$
\begin{gathered}
\text { Moscow, Russian Federation } \\
\text { e-mail: vilesin@inbox.ru }
\end{gathered}
$$

\begin{abstract}
Аннотация. Проведен анализ фрактальной теории с точки зрения соответствия ее выводов известным экспериментальным данным по изменению вязкости путем добавления химических соединений и воздействию физических полей. Дано обоснование использования химических реагентов различного типа, постоянных и переменных
\end{abstract}


электромагнитных полей и механических воздействий для снижения вязкости нефти путем разрушения агрегатов коллоидных частиц фрактального строения. Показано, что значительное снижение вязкости может быть достигнуто как результат полного разрушения фрактальных агрегатов на мелкие частицы, так и как результат формирования плотных фрактальных агрегатов, устойчивых к внешним воздействиям. На основе особенностей процессов разрушения и роста фрактальных агрегатов предложен общий подход к снижению вязкости путем варьирования во времени воздействия напряжением сдвига (скорости сдвига). Экспериментально продемонстрировано, что воздействием напряжения сдвига по предложенной в статье программе можно достичь многократного снижения вязкости и получить нефть, вязкость которой слабо зависит от скорости сдвига. Предложенный подход не требует получения информации о химическом составе нефти и базируется на измерении зависимости вязкости от скорости сдвига.

Abstract. An analysis of the fractal theory is based on the correspondence of its conclusions to the known experimental data on viscosity changes by adding chemical compounds and the effect of physical fields. The rationale for the use of chemical reagents of various types, constant and variable electromagnetic fields and mechanical stresses to reduce the oil viscosity by destroying aggregates of fractal structure colloidal particles is given. A general approach to reducing viscosity by varying the shear stress over time is proposed on the basis of the fracture and growth of fractal aggregates. It is experimentally demonstrated that by the influence of shear stress according to the program proposed in the article it is possible to achieve a multiple decrease in viscosity and obtain oil, the viscosity of which is weakly dependent on the shear rate. The proposed approach does not require information on the oil chemical composition and is based on measuring the dependence of viscosity on shear rate. 
Ключевые слова: нефть; вязкость; скорость сдвига; фрактальная теория; методика снижения вязкости; воздействие напряжением сдвига; коллоидные частицы

Key words: oil; viscosity; shear rate; fractal theory; viscosity reducing methods; shear stress effect; colloidal particles

\section{Введение}

Затраты энергии на перекачку в трубопроводах и на извлечение из горной породы возрастают с ростом вязкости нефти, поэтому разработка методов снижения вязкости является актуальной задачей для нефтегазовой отрасли.

Коллоидные жидкие растворы, в том числе нефть и нефтепродукты, как правило, обладают высокой вязкостью и проявляют свойства неньютоновских жидкостей, вязкость которых уменьшается под действием градиента скорости $V d V / d x=G$, где $G$ - скорость сдвига. В диапазоне больших значений $G$ (для нефти при комнатной температуре, как правило, это значение $G>10^{2} \mathrm{c}^{-1}$ ), когда вязкость очень слабо зависит от скорости сдвига, принято считать, что коллоидный раствор становится ньютоновской жидкостью.

Эйнштейном была рассмотрена задача об увеличении вязкости коллоидного раствора, содержащего низкие объемные концентрации сферических, абсолютно жестких, электрически нейтральных коллоидных частиц, взаимодействие между которыми не учитывалось. Согласно полученной формуле вязкость раствора не зависела от скорости сдвига и росла линейно с ростом объемной концентрации частиц.

Исследования реальных коллоидных растворов показали, что даже при объемных концентрациях порядка $10^{-5}-10^{-4} \%$ коллоидные частицы взаимодействуют между собой, что сопровождается образованием 
агрегатов фрактального строения, содержащих сотни-тысячи частиц. Кроме того, такие агрегаты не являются жесткими: они деформируются и разрушаются под действием напряжений, вызванных скоростью сдвига $[1,2]$, т.е. не выполняется второе условия, при котором коллоидный раствор имеет свойства ньютоновской жидкости.

Фрактальные агрегаты (ФА), образованные благодаря силам межмолекулярного взаимодействия, представляют механически непрочные структуры, которые могут разрушаться уже под действием напряжений, вызванных даже небольшими скоростями сдвига $G \approx 10^{-2}-10^{-1} \mathrm{c}^{-1}$ [3-5]. Появление таких коллективных структур коллоидных частиц проявляется в проявлении неньютоновских свойств коллоидных растворов.

Отличие неньютоновских жидких коллоидных растворов от ньютоновских состоит в проявлении взаимодействия между коллоидными частицами, что выражается в возникновении ФА, деформации и разрушении ФА при взаимодействии с вмещающей коллоидные частицы жидкостью. Приобретение раствором ньютоновских свойств создается при взаимодействии фрактальных агрегатов коллоидных частиц с вмещающей жидкостью.

Цель внесения химических реагентов, применяемых для снижения вязкости нефти, состоит в создании условий, препятствующих формированию коллективных коллоидных структур, уменьшению сил притяжения между коллоидными частицами и сохранению коллоидной составляющей в виде одиночных частиц $[6,7]$. Внесение и подбор таких реагентов определяются составом нефти и являются трудной задачей, осложняемой необходимостью применения реагентов, не вызывающих осложнений при переработке нефти. Типичные концентрации реагентов, как правило, составляют несколько десятков-сотен г на 1 т нефти.

Альтернативным способом снижения вязкости является гидродинамический [8], основанный на воздействии колебаниями 
давления, в том числе вызывающими кавитационные явления. Разработка научно обоснованных эффективных методов снижения вязкости нефти, основанных на гидродинамическом воздействии, является актуальной задачей, особенно в связи необходимостью замещения импорта химических реагентов,

В [9-11] предложена фрактальная теория, описывающая воздействие скоростью сдвига и электромагнитного поля на вязкость таких коллоидных растворов (систем), как нефть и нефтепродукты. Теория базируется на учете потерь гидродинамической энергии вмещающей жидкости на перемещение ФА, деформацию ФА, отрыв и присоединение коллоидных частиц к ФА. На примере нефти было показано соответствие теории известным особенностям эволюции вязкости неньютоновских растворов, предсказаны закономерности процессов релаксации вязкости при определенных режимах воздействия скорости сдвига и электромагнитного поля [10-13].

Благодаря интенсивным исследованиям физических свойств ФА $[1,2,14,15]$ установлены особенности изменения во времени их структуры и физико-химических свойств под действием температуры, скорости сдвига.

В предлагаемой работе рассмотрены методы снижения вязкости нефти и нефтепродуктов на основе фрактальной теории с использованием общих законов термодинамики для обоснования режимов воздействия скоростью сдвига (напряжением сдвига).

\section{Фрактальная теория о методах снижения вязкости}

Согласно фрактальной теории вязкости текущая вязкость коллоидного раствора [11]:

$$
\eta=\eta_{\infty}\left(1+K a^{3} n R^{\lambda}\right),
$$

где $R / a=R, R-$ радиус инерции ФА; 
$a$ - радиус коллоидной частицы;

$\lambda=\lambda(D)>0$ и $K=K(D)-$ константы, зависящие от фрактальной размерности массы $D$, где $D$ зависит от предыдущих физико-химических воздействий на коллоидную систему (коллоидный раствор);

$n$ - концентрация центров роста ФА;

$\eta_{\infty}-$ вязкость вмещающей жидкости.

При учете зависимости радиуса инерции $R$ от скорости сдвига $G$ : $R \sim\left(G_{d} / G\right)^{h}[1,2,13,14]$ стационарное значение вязкости, соответствующее данному значению $G$, имеет вид:

$$
\eta=\eta_{\infty}\left(1+K_{g} a^{3} n\left(G_{d} G^{\lambda h}\right)=\eta_{\infty}\left(1+B G^{-\alpha}\right),\right.
$$

где $\alpha=\lambda \mathrm{h}, \mathrm{B}=\mathrm{K}_{\mathrm{g}} \mathrm{a}^{3} \mathrm{n}\left(\mathrm{G}_{0}\right)^{\lambda \mathrm{h}}$.

Зависимость $\mathrm{R}$ от времени $\mathrm{t}$ описывается кинетическим уравнением, описывающим изменением массы $\mathrm{M} \sim \mathrm{R}^{\mathrm{D}}$ ФА от времени [11]:

$$
D R^{D-1} d R / d t=K_{1} R N-K_{2} G \eta_{\infty} a^{2} R^{p}-K_{3} R^{d},
$$

где $h>0, d>0, p>0, K_{g}$ и $G_{0}$ - константы, зависящие от фрактальной размерности $D$ и сил взаимодействия между частицами $\Phi А$;

$N$ - концентрация коллоидных частиц;

$K_{1}$ - константа реакции столкновения ФА и коллоидной частицы;

$K_{2}$ - константа реакции отрыва частиц от ФА под действием скорости сдвига $G$;

$K_{3}-$ константа реакции термического отрыва частиц от ФА.

ФА больших размеров $\mathrm{R}$ > 1 имеет проницаемую для жидкости слоистую, мультифрактальную фрактальную структуру $[1,4,9]$, которая выражается в том, что $\lambda(1 \leq \lambda \leq 3), h, K, K_{g}$ меняются в ходе разрушения или роста $R$ так, что $\lambda h_{i}=$ const и $K_{g}=$ const для $r_{i}<R<r_{i+1}$. Мультфрактальная структура ФА проявляется как зависимость стационарного значения $\operatorname{Ln}\left(\eta / \eta_{\infty}-1\right)$ от $\operatorname{Ln}\left(G_{0} / G\right)$ в виде ломаной линии, 
состоящей из соединенных между собой отрезков прямых, соответствующих определенным диапазонам значений $G[4,9,10,11]$.

Как следует из (1) и (2), если $R=1$ или $\lambda h=0$ (т.е. либо $\lambda=0$, либо $h=0$ ), то раствор становится ньютоновским, и формула (1) превращается в формулу Эйнштейна при $K g=5 / 2$. Это означает, что эти условия можно пренебречь процессами взаимодействия ФА с одиночными и малыми коллоидными частицами, не обладающими свойствами ФА. Параметр $\lambda$ представляет форм-фактор ФА, т.е. константа зависит от формы ФА, поэтому $\lambda>0$.

Согласно фрактальной теории добиться снижения вязкости нефти можно если:

$R \approx 1$, т.е. ФА полностью разрушены, и раствор содержит одиночные коллоидные частицы;

$h=0-$ коллоидные частицы в ФА связаны между собой достаточно большими силами, чтобы ФА не разрушался под действием напряжения сдвига.

При $G_{0} \rightarrow 0$ величина $G$, после достижения которой раствор становится ньютоновским, также приближается к 0, т.е. уже обеспечивая низкое значение $G$ можно получить ньютоновские свойства практически во всем диапазоне скоростей сдвига.

Из вышесказанного следует, что методы превращения неньютоновского раствора в ньютоновский состоят в формировании в нефти коллоидной системы, состоящей либо из одиночных коллоидных частиц, либо из плотных сферических ФА, непроницаемых для вмещающей жидкости. Достижение этих условий соответствует $d R / d t=0$, т.e. $K_{1}=K_{2}=K_{3}=0$.

Размер и, следовательно, динамическая вязкость $\eta$, определяются скоростями роста и разрушения ФА в результате прилипания и отрыва коллоидных частиц. При отрыве фрагмента ФА теряет энергию $\varepsilon_{1} \sim m G R^{2} / 2$, при прилипании частицы силы напряжения совершают работу 
по увеличению кинетической энергии коллоидной частицы от $m V^{2} / 2$ до энергии, соответствующей кинетической энергии на поверхности ФА $\varepsilon_{2} \sim m G R^{2} / 2$. Отрыв и прилипание сопровождаются потерей гидродинамической энергии жидкости, поскольку оторвавшаяся частица теряет свою кинетическую энергию, которая переходит в тепловую энергию раствора. Помимо отрыва-прилипания воздействие скорости сдвига приводит к деформации ФА в процессе вращения. При деформации - изменении формы, объема также происходят затраты гидродинамической энергии движения вмещающей жидкости на преодоление сил упругости, связанных с изменением энергии взаимодействия частиц в ФА и вытеснение вмещающей жидкости.

Вероятность отрыва фрагментов зависит от проницаемости поверхности ФА и сил взаимного притяжения частиц $[14,15]$. В предельном случае непрозрачной сферической поверхности затраты энергии на перемещение значительно уменьшаются и определяются силами вязкого трения жидкости на поверхность ФА. В этом случае вязкость будет определяться общим объемом коллоидных частиц и не зависеть от скорости сдвига.

Рассмотрим, каким путем можно реализовать оба метода на основе анализа процессов затрат гидродинамической энергии на процессы взаимодействия ФА и коллоидных частиц нефти.

Вклад ФА в стационарную вязкость раствора согласно определяется величиной $K_{g} a^{3} n\left(G_{d} / G\right)^{\lambda h}$. Сомножитель

$$
\left(\mathrm{G}_{0} / \mathrm{G}\right) \sim \mathrm{fa} / \mathrm{fc}
$$

где $f a-$ сила притяжения фрагмента ФА к поверхности;

$f_{c}$ - напряжение сдвига, действующего на фрагмент поверхности $[14,15]$.

Сила $f a$ определяется количеством контактов фрагмента и потенциалом взаимодействия $U$ между парами, который для коллоидных частиц 
сферической формы согласно теории ДЛФО может быть представлен в виде $U=U_{d i s p}+U_{e l}$, где потенциал $U_{d i s p}=-A a / 12 h$ вызван дисперсионными силами межмолекулярного притяжения (силами Ван-дер-Ваальса).

Сила притяжения $f a$ в положении потенциального минимума между парой частиц оценивается как $f a=A a / 12 h^{2}$, где А - эффективная константа Гамакера; $h$ - наименьшее расстояние между поверхностями частиц. Когда частица имеет нескольких близких соседей (число контактов $v_{c}$ ) потенциал $U_{\text {disp }}=-v_{c} A a / 12 h$, соответственно $f a \sim v_{\text {disp }} A a / 12 h^{2}$. Величина $v_{c}$ сложным образом растет с ростом фрактальной размерности массы ФА; при $D \approx 1 v_{c} \approx 2$, и ФА представляет линейно-цепочечную структуру [9, $10]$.

Для оценки $U_{e l}$ предложены формулы, которые предсказывают быстрое падение отталкивания частиц с ростом $h$, примем $U_{e l}=\beta e^{-\chi} h[16]$, где $\beta$ и $\chi$ - константы, зависящие от электрических зарядов частиц и диэлектрических свойств электролита, находящегося между частицами.

Суммируя потенциалы, получаем оценку

$$
\mathrm{U}=\beta \mathrm{e}^{-\mathrm{x}} \mathrm{h}-\mathrm{v}_{\mathrm{c}} \mathrm{Aa} / 12 \mathrm{~h},
$$

откуда сила притяжения частиц - минимум потенциала

$$
\mathrm{dU} / \mathrm{dh}=v_{\mathrm{c}} \mathrm{A} \text { a } / 12 \mathrm{~h}^{2 \cdot}-\chi \beta \mathrm{e}^{-\chi \mathrm{h}}=\mathrm{fa}=v_{\mathrm{c}}\left[(\mathrm{A}-\mathrm{E}) \mathrm{a} / 12 \mathrm{~h}^{2}\right] .
$$

Величина $f c \sim \eta_{\infty} G a^{2}[14,15]$, откуда

$$
\left(\mathrm{G}_{0} / \mathrm{G}\right) \sim \mathrm{fa} / \mathrm{fc} \sim v_{\mathrm{c}}\left[(\mathrm{A}-\mathrm{E}) / 12 \mathrm{~h}^{2} \eta_{\infty} \mathrm{Ga},\right.
$$

где $E$ - величина, пропорциональная $d U_{e l} / d h$;

$h$ - расстояние между поверхностями частиц в точке минимального значения потенциала $U$.

Константа $A$ оценивается, как правило, величиной порядка $10^{-14}-10^{-13}$ эрг [17].

При больших величинах заряда коллоидных частиц энергия электростатического отталкивания $U_{e l}$ может при всех расстояниях $h$ 
превышать энергию притяжения $U_{e l}>U_{d i s p .}$ В этом случае ФА не будут формироваться, дисперсная составляющая будет представлять одиночные частицы и плотные малые кластеры частиц, раствор будет ньютоновским. Отсюда следует метод использования ионогенных поверхностно-активных веществ (ПАВ), которые создают заряды у коллоидных частиц. Когда разница $(A-B)$ будет малой величиной, потребуются незначительные значения $G$ для разрушения ФА, и в этом случае раствор может приобрести ньютоновские свойства уже при значениях близких к нулевым.

Коллоидная частица нефти имеет структуру мицеллы, ядро которой размером 2-6 нм сформировано молекулами асфальтенов, окружено послойно молекулами смол, неполярных компонентов нефти типа масел, парафинов, циклических углеводородов и алканов на внешней границе частицы [6]. Уменьшить $f a$ можно за счет адсорбции на поверхности мицеллы ПАВ, обеспечивающих снижение эффективной константы $A$. Вклад в $A$ вносит слой небольшой толщины на поверхности коллоидной частицы: дисперсионные силы убывают как $\approx h^{-6}$, поэтому адсорбция даже нескольких слоев молекул ПАВ может существенно снизить $A$. Например, при замене толуола на гексан константа Гамакера для взаимодействия смолисто-асфальтеновых частиц уменьшается более, чем в три раза [18].

Увеличение концентрации центров роста ФА $n$, которыми являются магнитные коллоидные наночастицы оксида железа и их кластеры [19], также приводит к снижению вязкости $[11,20,21]$. В $[22,23]$ обнаружено, что обработка магнитным полем нефти приводит как к диспергированию коллоидной составляющей нефти - уменьшению радиусов частиц нефти, так и многократному снижению вязкости при малых скоростях сдвига [22]. Дробление ФА под действием магнитного поля происходит благодаря присутствию в составе ФА магнитных частиц оксидов железа [9-11]. Снижение вязкости нефти происходит благодаря тому, что ФА малого размера обладают высокой плотностью и малой пористостью и имеют 
небольшой рыхлый внешний слой, который разрушается уже при относительно малых значениях $G$.

Согласно формуле (1) снижение вязкости связано с уменьшением величины произведения $K a^{3} n R^{\lambda}$, которое линейно зависит от $n$ и степенным образом зависит от $R$. В [11] на основе анализа экспериментальных данных показано, что рост $n$ приводит к снижению вязкости неньютоновской жидкости. Как следует из вышесказанного, наиболее значительное снижение вязкости после магнитной обработки наблюдается в области малых значений $G$, где вязкость сильно зависит от скорости сдвига. Размеры ФА и вязкость после магнитной обработки медленно увеличиваются, достигая предельной величины за время порядка 10-20 ч [22], что соответствует агрегации фрагментов ФА, возникших после магнитной обработки.

Оценка сил взаимного отталкивания в результате ориентации магнитных моментов вдоль внешнего магнитного поля была произведена на основе учета типичных размеров стержнеобразных магнитных частиц [23, 24], значений постоянной Гамакера и магнитных свойств оксидов железа. Оценка показала, что во внешнем достаточно сильном магнитном поле порядка 0,1-0,2 Тл силы магнитного расталкивания $\approx 3 \cdot 10^{-3}$ дин уже достаточны для разделения магнитных частиц, поскольку многократно превышают силы их дисперсионного притяжения $\approx 3 \cdot 10^{-5}$ дин.

Максимальный эффект снижения вязкости и темпов образования асфальтосмолопарафиновых отложений в области температур и давлений достигается магнитной обработкой до начала процесса образования коллоидных частиц нефти, поэтому магнитные устройства для предотвращения отложений парафина устанавливаются в скважине ниже области начала появления отложений [25]. Эффективность обработки магнитным полем уже сформировавшейся коллоидной дисперсной системы снижается, поскольку в этом случае силы магнитного отталкивания должны превосходить не только силы межмолекулярного 
притяжения частиц оксидов железа, но и силы притяжения коллоидных частиц нефти.

Поскольку коллоидные магнитные частицы оксида железа, образующие ФА, обладают электрическими зарядами (положительными зарядами в слабокислой среде - нефти) возможно разрушение ФА достаточно сильным электрическим полем, вызывающим движение частиц железа в ФА и отрыв от ФА фрагментов. Воздействие электрического поля на неньтоновскую нефть также сопровождается снижением вязкости на десятки процентов [26] поскольку воздействие электрическим полем аналогично воздействию магнитным полем.

Действительно, сила, действующая на коллоидную частицу нефти, равна $E q$ ( $E$ - напряженность электрического поля; $q$ - заряд частицы). При типичном значении $E=10^{6} \mathrm{~B} / \mathrm{M}$ [26] и заряде частицы $q \approx 10^{-14}$ Кл (примерно $10^{5}$ зарядов электрона) сила электрического воздействия составит $10^{-3}$ дин и превысит силу притяжения между противоположно заряженными коллоидной частицей нефти и коллоидной частицей оксида железа $\approx 10^{-5}$ дин, вызвав дробление $Ф А$, как и в случае магнитной обработки.

Использование ПАВ требует подбора состава и концентрации химреагентов, учитывающих вещественный состав конкретной нефти, что представляет сложную научную задачу. Использование магнитной обработки требует присутствия магнитных частиц в составе нефти, что может быть обеспечено естественными примесями, концентрация которых, как правило, составляет несколько десятков г на 1 т, и меняется в широком диапазоне в зависимости от месторождения и конкретной скважины или трубопровода. Кроме того, вязкость нефти после магнитной обработки, как правило, возрастает до величины, близкой первоначальной. Внесение частиц извне представляет сложную техническую задачу, требующую создания устройств внесения и распределения частиц по объему нефти. 
Рассмотрим на основе фрактальной теории вопрос о такой методике снижения вязкости путем воздействия скоростью сдвига, которая опирается на измерения вязкости и не требует определения состава нефти, нефтепродуктов.

Из исследований ФА известно, что длительное воздействие скоростью сдвига сопровождается ростом фрактальной размерности $D$, которая характеризует плотность ФА [14, 15] Масса ФА связана с радиусом инерции $R$ и $D$ соотношением $M \sim R^{D}, D=3$ соответствует максимальной плотности упаковки частиц, что соответствует максимальной устойчивости ФА по отношению к процессам разрушения. Коллоидный раствор с ФА, имеющими размерность $D=3$, приближается по свойствам вязкости к ньютоновским жидкостям и имеет наименьшую вязкость при данном объеме коллоидных частиц.

При разработке программь воздействия скорости сдвига с целью снижения вязкости покоящейся нефти необходимо учитывать следующее.

1. При столкновениях с ФА коллоидная частица может первоначально занять не самое выгодное с энергетической точки зрения, однако достаточно устойчивое положение локального минимума свободной энергии. Для занятия положения, при котором энергия связи частица-ФА максимальна, необходима деформация или перемещение частицы внутри ФА, что требует преодоления потенциального барьера - дополнительных затрат энергии для перестройки структуры ФА, соответствующей новой величине свободной энергии. Это проявляется в зависимости физикохимических характеристик нефти и нефтепродуктов от истории предшествующих воздействий температурой и напряжением сдвига [13, $27,28]$.

2. После воздействия достаточно больших значений $G$, приведших к разрушению ФА, чтобы получить маловязкую нефть в состоянии покоя при $G=0$, необходимо снижать $G$ таким образом, чтобы в ходе роста ФА представляли плотную, шарообразную, малопористую структуру. Из 
фрактальной теории следует, что ФА увеличиваются в размерах, когда закрепление отдельной коллоидной или малой частицы (кластера частиц) происходит лишь в случаях, когда сила притяжения коллоидной частицы к ФА (количество контактов с соседями) достаточно большое и сила напряжения сдвига $G a^{2} \eta_{\infty}$ недостаточна для отрыва коллоидной частицы, что соответствует $f a>G a^{2} \eta_{\infty}$.

Для создания таких условий необходимо предварительно разрушить ФА с целью получения одиночных, или плотных, достаточно малых частиц $(R<10 a)$, не обладающих фрактальными свойствами. Это возможно в случае, когда режим увеличения $G$ подбирается таким образом, чтобы переход к новому значении происходил после достижения стационарного значения вязкости $\eta$ при каждом новом значении $G$. После достижения конечного, достаточно большого значения $G$, когда $\eta \approx$ const, нужно сразу переходить к снижению $G$ в условиях достижения термодинамического равновесия при каждом значении $G$. Такой режим изменения напряжения сдвига позволит сформировать дисперсную систему в виде устойчивых к разрушению плотных ФА.

С точки зрения фрактальной теории это означает, что менять во времени величину $G$ нужно таким образом, чтобы $d R / d t \approx 0$ и $\alpha=\lambda h \approx 0$. Быстрое изменение скорости сдвига будет приводить к закреплению на ФА как отдельных коллоидных частиц, так и малых плотных кластеров, состоящих из нескольких частиц, на поверхности агрегата в положении, не всегда соответствующем максимальной энергией связи с ФА.

Для подтверждения возможности создания такого режима воздействия был проведен эксперимент, в котором на первоначально покоившийся прямогонный мазут, использовавшийся в качестве модели тяжелой нефти, воздействовали скоростью сдвига, повышая ступенчато $G$ от $0,1 \mathrm{c}^{-1}$ до $10^{3} \mathrm{c}^{-1}$, переход к новому значению $G$ осуществлялся через время, когда устанавливалось стационарное значение вязкости на предыдущем 
значении $G$. После достижения $G=10^{3} \mathrm{c}^{-1}$ скорость сдвига сразу ступенчато снижалась до $G=0,1 \mathrm{c}^{-1}$.

На рисунке 1 приведена зависимость $\eta(G)$, зарегистрированная в ходе эксперимента по снижению вязкости прямогонного мазута путем формирования плотных ФА, устойчивых к механическому воздействию. Общее время воздействия скоростью сдвига составило 90 мин (45 мин роста $G$ плюс 45 мин снижения). Величина вязкости фиксировалась при каждом новом значении $G$ при достижении постоянного значения величины вязкости, т.е. при достижении равновесия в коллоидной системе.

$\eta, \Pi a^{\cdot} \mathrm{c}$

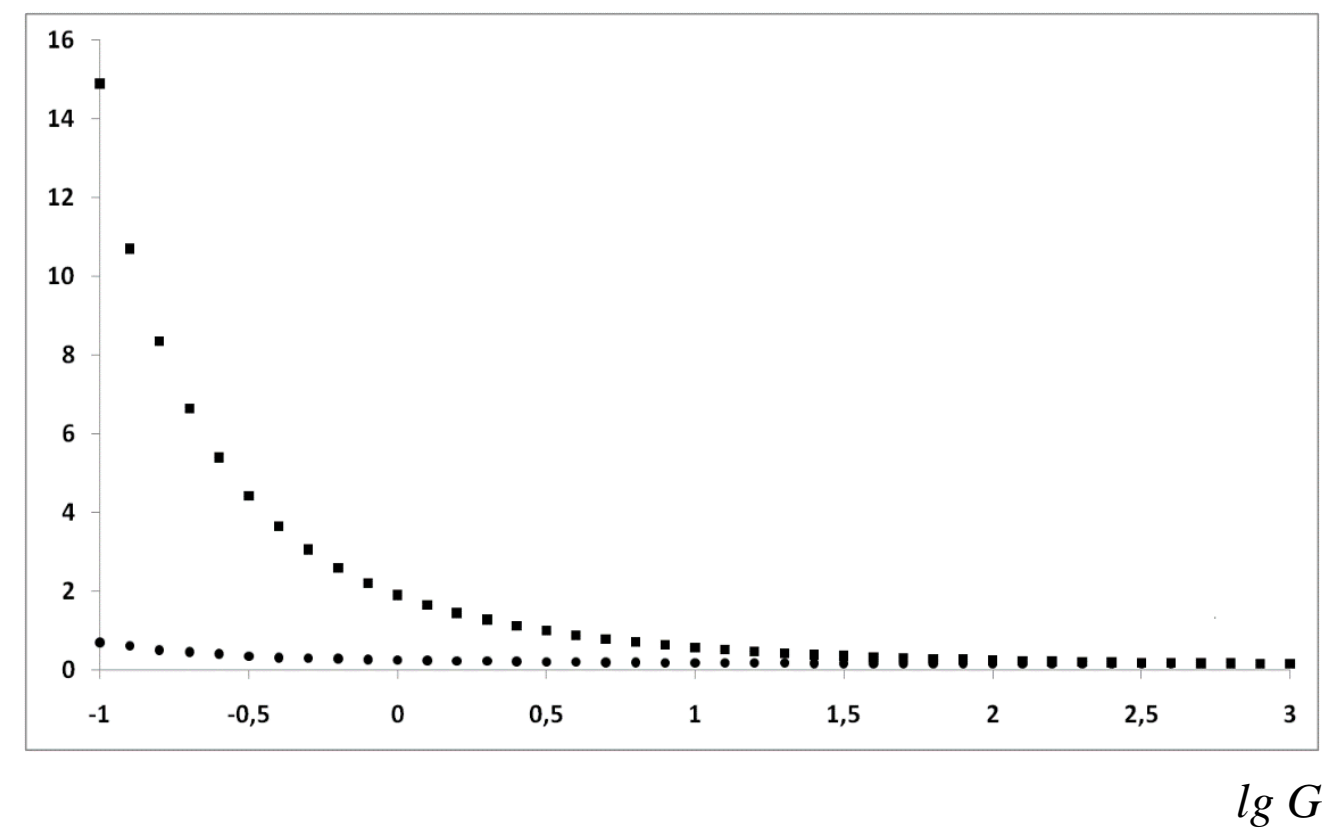

- (верхний ряд точек) - рост $G$;

- (нижний ряд точек) - снижение $G$

Рисунок 1. Зависимость вязкости $\eta$ от скорости сдвига в ходе роста и снижения $\mathrm{G}$

Зависимость вязкости от скорости сдвига описывалась формулой $\eta=\eta_{\infty}\left(1+B G^{-\alpha}\right)$ в ходе роста и снижения $G$, где $\eta_{\infty}=0,11$ Па $\cdot$. При росте $G$ величина $\alpha=1$ в диапазоне $0,1 \mathrm{c}^{-1} \leq G \leq 0,4 \mathrm{c}^{-1}$ снизилась до $\alpha=0,2$ в диапазоне $160 \mathrm{c}^{-1} \leq G \leq 1000 \mathrm{c}^{-1}$. Величина $\alpha=0$ соответствует 
ньютоновской жидкости, вязкость которой не зависит от скорости сдвига. Величина $\alpha=0,2$ показывает, что под действием напряжений сдвига в этом диапазоне ФА продолжался процесс роста-разрушения и деформации ФА. При снижении $G$ в диапазоне $6 \mathrm{c}^{-1} \leq G \leq 1000 \mathrm{c}^{-1}$ величина $\alpha=0,05 \approx 0$, что свидетельствует о том, что в этом случае сформировались плотные ФА, устойчивые к воздействию напряжения сдвига, т.е. была достигнута поставленная цель. Дальнейшее снижение $G$ сопровождалось ростом $\alpha$ до 0,6 в диапазоне $0,1 \mathrm{c}^{-1} \leq G \leq 0,3 \mathrm{c}^{-1}$, что свидетельствует о формировании слоя частиц, слабо связанных с поверхностью ФА. Достигнутая в результате обработки сдвигом вязкость 0,66 Па·с при $G=0,1 \mathrm{c}^{-1}$ более чем в 20 раз $(15$ Па с $)$ меньше исходной вязкости мазута.

На рисунке 2 приведены результаты измерения напряжения сдвига $\tau=\eta G=\eta_{\infty}\left(G+B G^{1-\alpha}\right)$.

$\tau, \Pi \mathrm{a}$

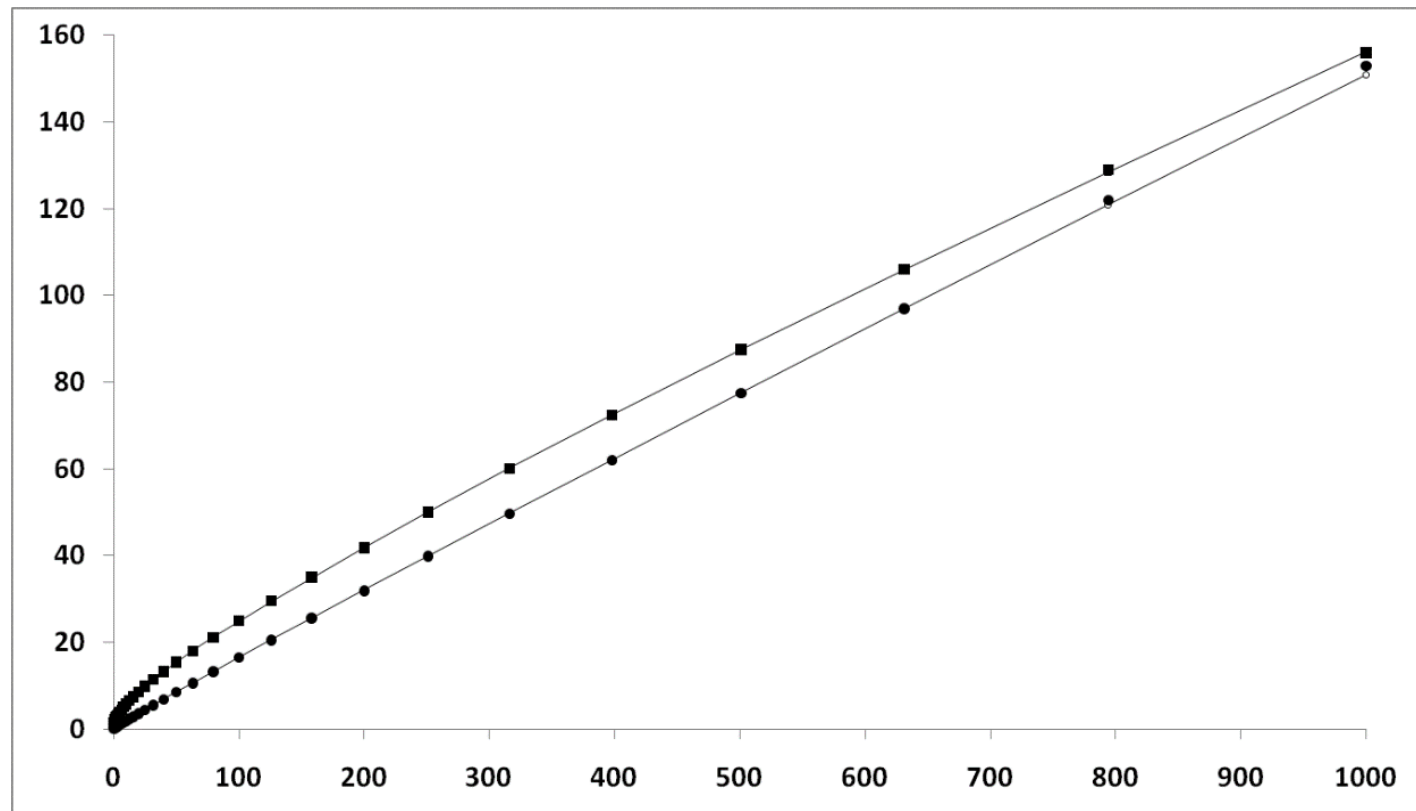

$\mathrm{G}, \mathrm{c}^{-1}$

- (верхний ряд) - экспериментальные данные, полученные в ходе роста $\mathrm{G}$;

- (нижний ряд) - экспериментальные данные, полученные в ходе снижения $\mathrm{G}$; сплошные линии - расчет по формуле $\tau=\eta_{\infty}\left(G+B G^{l-\alpha}\right)$, где $\eta_{\infty}=0,11(\Pi a \cdot c), \eta_{\infty}, B$ и $\alpha$ определены из экспериментальных данных

Рисунок 2. Зависимость напряжения сдвига $\tau=\eta G$ от скорости сдвига $G$, полученная в ходе эксперимента по снижению вязкости 
Из рисунка 2 видно, что после обработки напряжение сдвига почти линейно зависит от скорости сдвига практически во всем диапазоне $G$, поскольку величина $\alpha \approx 0$ вплоть до $G \approx 6 c^{-1}$. Величина относительного снижения напряжения сдвига до обработки скоростью сдвига была выше значения после обработки на $\approx 70 \%$ при $G=100 \mathrm{c}^{-1}$ и на $\approx 300 \%$ и выше в диапазоне $G \leq 10 \mathrm{c}^{-1}$.

Поскольку при $\alpha \approx 0$ напряжение сдвига $\tau \approx \eta_{\infty}(1+B) G$, мы получаем ньютоновские свойства вязкой среды в данном диапазоне значений $G$. Согласно формуле Эйнштейна для сферических жестких частиц в этом случае $B=(5 / 2) v_{\text {coll }} / v$, где $v_{\text {coll }}$ - общий объем коллоидных частиц нефти, $v$ - общий объем коллоидного раствора). Поскольку $v_{\text {coll }} / v<1$, то после воздействие скоростью сдвига позволяет получить коллоидный раствор, вязкость которого примерно в 3,5 раза превышает вязкость жидкой составляющей нефти. Для модельного образца нефти - мазута в области $6,31 \mathrm{c}^{-1} \leq G \leq 1000 \mathrm{c}^{-1}$, где вязкость соответствует ньютоновской жидкости $(\alpha=0,05 \approx 0)$ величина $B=3$, что близко к 2,5, и вязкость примерно в 4 раза превышает вязкость $\eta_{\infty}$ вмещающей жидкости.

Как видно из полученных результатов, обработанный коллоидный раствор будет оказывать значительно меньшее гидравлическое сопротивление, чем необработанный, за счет снижения вязкости в наиболее важном диапазоне скоростей сдвига. Это является одной из причин роста дебита нефти при обработке коллоидного раствора нефти колебаниями давления. Вязкость воды, которая является ньютоновской жидкостью, не меняется, поэтому снижается относительная доля воды в продукции скважины.

\section{Выводы}

Анализ фрактальной теории, основанной на физических процессах взаимодействия коллоидных частиц между собой и вмещающей жидкостью, показал соответствие теории наблюдаемым изменениям 
вязкости, возникающим под действием реагентов и физико-механических воздействий на дисперсную систему нефти и нефтепродуктов. Знание физических процессов, лежащих в основе проявления неньютоновских свойств коллоидных растворов, дает возможность разрабатывать методы воздействия на вязкость нефти и нефтепродуктов, не прибегая к использованию химических соединений.

Статья написана в рамках выполнения государственного задания (тема «Исследование термодинамических свойств углеводородных смесей, моделирование гидротермодинамических, фризико-химических и геомеханических процессов в геосредах с изелью повымения эффективности разработки трудноизвлекаемых запасов нефти и газа», № AAAA-A19-119030690057-5).

\section{Список используемых источников}

1. Roldughin V.I. The Characteristics of Fractal Disperse System // Russian Chemical Review. 2003. Vol. 72. No. 11. P. 913-937. DOI: 10.1070/RC2003v072n11ABEH000829.

2. Bushella G.C., Yan Y.D., Woodfield D., Raper J., Amal R. On Techniques for the Measurement of the Mass Fractal Dimension of Aggregates // Advances in Colloid and Interface Science. 2002. Vol. 95. P. 1-50. DOI: 10.1016/s0001-8686(00)00078-6.

3. Лесин В.И., Лесин С.В. Фрактальная теория и экспериментальные исследования вязкости коллоидных систем при скоростях сдвига, близких к нулю // Нефтяное хозяйство. 2013. № 7. С. 111-113.

4. Лесин В.И., Клепиков И.А. Применение фрактальной теории вязкости дисперсных систем к аномальной зависимости вязкости от скорости сдвига // Нефтяное хозяйство. 2015. № 2. С. 38-41. 
5. Лесин В.И., Лесин С.В. Влияние колебаний давления на вязкость нефти, содержащей коллоидные частицы // Научное сетевое издание «Актуальные проблемы нефти и газа». 2019. Вып. 1 (24). URL: http://oilgasjournal.ru/issue_24/lesin.html (дата обращения: 20.08.2019). DOI: 10.29222/ipng.2078-5712.2019-24.art11.

6. Сюняев З.И., Сафиева Р.З., Сюняев Р.З. Нефтяные дисперсные системы. М.: Химия, 1990. 226 с.

7. Башкирцева Н.Ю., Сладовская О.Ю. Особенности применения ПАВ в процессах транспортировки высоковязких нефтей // Вестник технологического университета. 2014. Т. 17. Вып. 14. С. 449-451.

8. Ершов М.А., Баранов Д.А., Муллакаев М.С. Снижение вязкости парафинистых нефтей обработкой в гидродинамическом реакторе // Химическое и нефтегазовое машиностроение. 2011. № 7. С. 16-19.

9. Lesin V.I., Koksharov Yu.A., Khomutov G.B. Viscosity of Liquid Suspensions with Fractal Aggregates: Magnetic Nanoparticles in Petroleum Colloidal Structures // Colloids and Surfaces A Physicochemical and Engineering Aspects. 2011. Vol. 392. P. 88-94. DOI: 10.1016/j.colsurfa.2011.09.038.

10. Лесин В.И., Лесин С.В. Фрактальная формула зависимости вязкости неньютоновской жидкости от градиента скорости // Нефтяное хозяйство. 2012. № 3. С. 46-48.

11. Лесин В.И. Математическая модель вязкости тяжелой нефти, содержащей примеси коллоидных наночастиц оксидов металлов // Сетевое издание «Нефтегазовое дело». 2019. № 2. С. 200-227. URL: http://ogbus.ru/files/ogbus/issues/2_2019/ogbus_2_2019_p199-216.pdf (дата обращения: 13.08.2019). DOI: 10.17122/ogbus-2019-2-199-216.

12. Лесин В.И. Агрегация коллоидных частиц, регистрируемая по изменению вязкости // Научное сетевое издание «Актуальные проблемы нефти и газа». 2016. Вып. 2 (14). URL: http://www.oilgasjournal. ru/issue_14/lesin.html (дата обращения: 22.08.2019). DOI: 10.29222/ipng.2078-5712.2016-14.art16. 
13. Лесин В.И., Алексеева Ю.В. Эволюция структуры фрактальных агрегатов нефти под действием напряжения сдвига // Научное сетевое издание «Актуальные проблемы нефти и газа». 2018. Вып. 3 (22). URL: http://oilgasjournal.ru/issue_22/lesin.html (дата обращения: 12.08.2019). DOI: 10.29222/ipng.2078-5712.2018-22.art28.

14. Sonntag R.C., Russel W.B. Structure and Breakup of Flocs Subjected to Fluid Stresses.1. Shear Experiments // Journal of Colloid and Interface Science. 1986. Vol. 113. P. 399-413.

15. Sonntag R.C., Russel W.B. Structure and Breakup of Flocs Subjected to Fluid Stresses.1. Theory // Journal of Colloid and Interface Science. 1987. Vol. 115. No. 2. P. 378-389.

16. Guozhong C., Ying W. Nanostructures and Nanomaterials: Synthesis, Properties, and Applications. Singapore: World Scientific Publishing Company, 2004. 596 p.

17. Лесин В.И., Лесин С.В. Физико-химический механизм воздействия колебаний давления жидкости на фильтрационные свойства нефти и пористой среды // Бурение и нефть. 2003. № 1. С. 24-27.

18. Яковец Н.В., Опанасенко О.Н., Крутько Н.П. Модифицирование нефтяных смолисто-асфальтеновых дисперсий оксиэтилированными поверхностно-активными веществами // Весци нацыональной академии навук Беларуси, серыя химичных навук. 2013. № 2. С. 10-15.

19. Pastor-Satorras R., Rubi J.M. Fractal Properties of Cluster of Colloidal Magnetic Particles // Progress in Colloid and Polymer Science. 1998. Vol. 110. P. 29-33. DOI: 10.1007/BFb0118043.

20. Aristizabal-Fontal J.E., Cortes F.B., Franco C.A. Viscosity Reduction of Extra Heavy Crude Oil by Magnetite Nanoparticle-Based Ferrofluids // Adsorption Science and Technology (Special Collection: III Workshop on Adsorption, Catalysis and Porous Materials). 2017. P. 1-23. DOI: $10.1177 / 0263617417704309$. 
21. Nassar N.N, Betancur S., Acevedo S. Development of a Population Balance Model to Describe the Influence of Shear and Nanoparticles on the Aggregation and Fragmentation of Asphaltene Aggregates // Industrial and Engineering Chemistry Research. 2015. Vol. 54. P. 8201-8211.

22. Лоскутова Ю.В., Юдина Н.В. Влияние постоянного магнитного поля на реологические свойства высокопарафинистых нефтей // Коллоидный журнал. 2003. Т. 65. № 4. С. 510-515.

23. Evdokimov I.N., Kornishin K.A. Apparent Disaggregation of Colloids in a Magnetically Treated Crude Oil // Energy and Fuels. 2009. Vol. 23. Issue 8. P. 4016-4020. DOI: 10.1021/ef900296e.

24. Лесин В.И. Физико-химические основы нетеплового воздействия электромагнитных и акустических полей на нефть для предотвращения отложений парафина // Нефтяное хозяйство. 2004. № 1. С. 68-70.

25. Лесин В.И. Область наиболее эффективного применения магнитных депарафинизаторов при защите от отложений насоснокомпрессорных труб добывающих скважин // Бурение и нефть. 2003. № 1. C. 24-27.

26. Tao R., Tang H. Reducing Viscosity of Paraffin Based Crude Oil with Electric Field for Oil Production and Transportation // Fuel. 2014. Vol. 118. P. 69-72. DOI: 10.1016/j.fuel.2013.10.056.

27. Евдокимов И.Н., Елисеев Н.Ю. Долгоживущие метастабильные состояния коллоидных структур нефтяных остатков // Химия и технология топлив и масел. 2005. № 3. С. 45-47.

28. Лесин В.И., Еремин Н.А. Природные и синтезированные наноразмерные окислы железа - нанороботы в процессах управления с помощью магнитного поля извлечением, транспортировкой, подготовкой и переработкой нефти // Нефть. Газ. Новации. 2018. № 1. С. 18-22. 


\section{References}

1. Roldughin V.I. The Characteristics of Fractal Disperse System. Russian Chemical Review, 2003, Vol. 72, No. 11, pp. 913-937. DOI: 10.1070/RC2003v072n11ABEH000829.

2. Bushella G.C., Yan Y.D., Woodfield D., Raper J., Amal R. On Techniques for the Measurement of the Mass Fractal Dimension of Aggregates. Advances in Colloid and Interface Science, 2002, Vol. 95, pp. 1-50. DOI: 10.1016/s0001-8686(00)00078-6.

3. Lesin V.I., Lesin S.V. Fraktal'naya teoriya i eksperimental'nye issledovaniya vyazkosti kolloidnykh sistem pri skorostyakh sdviga, blizkikh k nulyu [Fractal Theory and Experimental Research of Colloidal Systems Viscosity at Shear Rates Close to Zero Value]. Neftyanoe khozyaistvo - Oil Industry, 2013, No. 7, pp. 111-113. [in Russian].

4. Lesin V.I., Klepikov I.A. Primenenie fraktal'noi teorii vyazkosti dispersnykh sistem $\mathrm{k}$ anomal'noi zavisimosti vyazkosti ot skorosti sdviga [Fractal Viscosity Theory Application to Anomalous Viscosity Dependence on Share Rate]. Neftyanoe khozyaistvo - Oil Industry, 2015, No. 2, pp. 38-41. [in Russian].

5. Lesin V.I., Lesin S.V. Vliyanie kolebanii davleniya na vyazkost' nefti, soderzhashchei kolloidnye chastitsy [Influence of Pressure Fluctuations on the Viscosity of Oil Containing Colloidal Particles]. Nauchnoe setevoe izdanie «Aktual'nye problemy nefti i gaza»-Actual Problems of Oil and Gas, 2019, Issue 1 (24). Available at: http://oilgasjournal.ru/issue_24/lesin.html (accessed 20.08.2019). DOI: 10.29222/ipng.2078-5712.2019-24.art11 [in Russian].

6. Syunyaev Z.I., Safieva R.Z., Syunyaev R.Z. Neftyanye dispersnye sistemy [Oil Disperse Systems]. Moscow, Khimiya Publ, 1990. 226 p. [in Russian].

7. Bashkirtseva N.Yu., Sladovskaya O.Yu. Osobennosti primeneniya PAV $\mathrm{v}$ protsessakh transportirovki vysokovyazkikh neftei [Features of Application of Surfactants in the Process of Transportation of High-Viscosity Oils]. Vestnik tekhnologicheskogo universiteta - Herald of Kazan Technological University, 2014, Vol. 17, Issue 14, pp. 449-451. [in Russian]. 
8. Ershov M.A., Baranov D.A., Mullakaev M.S. Snizhenie vyazkosti parafinistykh neftei obrabotkoi $\mathrm{v}$ gidrodinamicheskom reaktore [Reducing the Viscosity of Paraffin Oil Treatment in a Hydrodynamic Reactor]. Khimicheskoe i neftegazovoe mashinostroenie - Chemical And Petroleum Engineering, 2011, No. 7, pp. 16-19. [in Russian].

9. Lesin V.I., Koksharov Yu.A., Khomutov G.B. Viscosity of Liquid Suspensions with Fractal Aggregates:Magnetic Nanoparticles in Petroleum Colloidal Structures. Colloids and Surfaces A Physicochemical and Engineering Aspects, 2011, Vol. 392, pp. 88-94. DOI: 10.1016/j.colsurfa.2011.09.038.

10. Lesin V.I., Lesin S.V. Fraktal'naya formula zavisimosti vyazkosti nen'yutonovskoi zhidkosti ot gradienta skorosti [Fractal Formula of NonNewtonian Fluid Viscosity Dependence on Shear Rate]. Neftyanoe khozyaistvo Oil Industry, 2012, No. 3, pp. 46-48 [in Russian].

11. Lesin V.I. Matematicheskaya model' vyazkosti tyazheloi nefti, soderzhashchei primesi kolloidnykh nanochastits oksidov metallov [Viscosity Mathematical Model of Heavy Oil Containing the Metal Oxides Colloid Nanoparticles Impurity]. Setevoe izdanie «Neftegazovoe delo» - Online Edition «Oil and Gas Business», 2019, No. 2, pp. 200-227. URL: http://ogbus.ru/files/ogbus/issues/2_2019/ogbus_2_2019_p199-216.pdf (accessed 13.08.2019). DOI: 10.17122/ogbus-2019-2-199-216 [in Russian].

12. Lesin V.I. Agregatsiya kolloidnykh chastits, registriruemaya po izmeneniyu vyazkosti [Aggregation of Colloidal Particles Registered by Viscosity Change Measurements]. Nauchnoe setevoe izdanie "Aktual'nye problemy nefti i gaza» - Actual Problems of Oil and Gas, 2016, Issue 2 (14). Available at: http://www.oilgasjournal.ru/issue_14/lesin.html (accessed 22.08.2019). DOI: 10.29222/ipng.2078-5712.2016-14.art16 [in Russian].

13. Lesin V.I., Alekseeva Yu.V. Evolyutsiya struktury fraktal'nykh agregatov nefti pod deistviem napryazheniya sdviga [Evolution of the Structure of Oil Fractal Aggregates under the Action of Shear Stress]. Nauchnoe setevoe izdanie «Aktual'nye problemy nefti i gaza» - Actual Problems of Oil and Gas, 2018, Issue 
3 (22). Available at: http://oilgasjournal.ru/issue_22/lesin.html (accessed 12.08.2019). DOI: 10.29222/ipng.2078-5712.2018-22.art28 [in Russian].

14. Sonntag R.C., Russel W.B. Structure and Breakup of Flocs Subjected to Fluid Stresses.1. Shear Experiments. Journal of Colloid and Interface Science, 1986, Vol. 113, pp. 399-413.

15. Sonntag R.C., Russel W.B. Structure and Breakup of Flocs Subjected to Fluid Stresses.1. Theory. Journal of Colloid and Interface Science, 1987, Vol. 115, No. 2, pp. 378-389.

16. Guozhong C., Ying W. Nanostructures and Nanomaterials: Synthesis, Properties, and Applications. Singapore, World Scientific Publishing Company, 2004. 596 p.

17. Lesin V.I., Lesin S.V. Fiziko-khimicheskii mekhanizm vozdeistviya kolebanii davleniya zhidkosti na fil'tratsionnye svoistva nefti i poristoi sredy [Physico-Chemical Mechanism of Influence of Liquid Pressure Fluctuations on Filtration Properties of Oil and Porous Medium]. Burenie i neft' - Drilling and Oil, 2003, No. 1, pp. 24-27. [in Russian].

18. Yakovets N.V., Opanasenko O.N., Krutko N.P. Modifitsirovanie neftyanykh smolisto-asfal'tenovykh dispersii oksietilirovannymi poverkhnostnoaktivnymi veshchestvami [Modification of Oil Resin-Asphaltene Dispersions by Oxyethylated Surfactants]. Vestsi natsyonal'noi akademii navuk Belarusi, seryya khimichnykh navuk - Proceedings of the National Academy of Sciences of Belarus. Chemical Series, 2013, No. 2, pp. 10-15. [in Russian].

19. Pastor-Satorras R., Rubi J.M. Fractal Properties of Cluster of Colloidal Magnetic Particles. Progress in Colloid and Polymer Science, 1998, Vol. 110, pp. 29-33. DOI: 10.1007/BFb0118043.

20. Aristizabal-Fontal J.E., Cortes F.B., Franco C.A. Viscosity Reduction of Extra Heavy Crude Oil by Magnetite Nanoparticle-Based Ferrofluids. Adsorption Science and Technology (Special Collection: III Workshop on Adsorption, Catalysis and Porous Materials), 2017, pp. 1-23. DOI: $10.1177 / 0263617417704309$. 
21. Nassar N.N, Betancur S., Acevedo S. Development of a Population Balance Model to Describe the Influence of Shear and Nanoparticles on the Aggregation and Fragmentation of Asphaltene Aggregates. Industrial and Engineering Chemistry Research, 2015, Vol. 54, pp. 8201-8211.

22. Loskutova Yu.V., Yudina N.V. Vliyanie postoyannogo magnitnogo polya na reologicheskie svoistva vysokoparafinistykh neftei [Influence of a Constant Magnetic Field on Rheological Properties of High-Paraffin Oils]. Kolloidnyi zhurnal - Colloid Journal, 2003, Vol. 65, No. 4, pp. 510-515. [in Russian].

23. Evdokimov I.N., Kornishin K.A. Apparent Disaggregation of Colloids in a Magnetically Treated Crude Oil. Energy and Fuels, 2009, Vol. 23, Issue 8, pp. 4016-4020. DOI: 10.1021/ef900296e.

24. Lesin V.I. Fiziko-khimicheskie osnovy neteplovogo vozdeistviya elektromagnitnykh i akusticheskikh polei na neft' dlya predotvrashcheniya otlozhenii parafina [Physico-Chemical Bases of Non-Thermal Influence of Electromagnetic and Acoustic Fields on Oil to Prevent Paraffin Deposits]. Neftyanoe khozyaistvo - Oil Industry, 2004, No. 1, pp. 68-70. [in Russian].

25. Lesin V.I. Oblast' naibolee effektivnogo primeneniya magnitnykh deparafinizatorov pri zashchite ot otlozhenii nasosno-kompressornykh trub dobyvayushchikh skvazhin [The Field of the Most Effective Application of Magnetic Dewaxing Agents for Protection Against Deposits of Pump and Compressor Pipes of Producing Wells]. Burenie i neft ${ }^{\prime}$ - Drilling and Oil, 2003, No. 1, pp. 24-27. [in Russian].

26. Tao R., Tang H. Reducing Viscosity of Paraffin Based Crude Oil with Electric Field for Oil Production and Transportation. Fuel, 2014, Vol. 118, pp. 69-72. DOI: 10.1016/j.fuel.2013.10.056.

27. Evdokimov I.N., Eliseev N.Yu. Dolgozhivushchie metastabil'nye sostoyaniya kolloidnykh struktur neftyanykh ostatkov [Long-Lived Metastable States of Colloidal Structures of Oil Residues]. Khimiya i tekhnologiya topliv $i$ masel - Chemistry and Technology of Fuels and Oils, 2005, No. 3, pp. 45-47. [in Russian]. 
28. Lesin V.I., Eremin N.A. Prirodnye i sintezirovannye nanorazmernye okisly zheleza - nanoroboty $\mathrm{v}$ protsessakh upravleniya $\mathrm{s}$ pomoshch'yu magnitnogo polya izvlecheniem, transportirovkoi, podgotovkoi i pererabotkoi nefti [The Natural and Synthesized Nanoscale Iron Oxides - Nanobots in the Control Processes of the Production, the Transportation, the Preparation and the Refining of Oil by Using the Magnetic Field]. Neft'. Gaz. Novatsii-Oil. Gaz. Novation, 2018, No. 1, pp. 18-22. [in Russian].

\section{Сведения об авторах}

\section{About the authors}

Лесин Виктор Иванович, канд. физ.-мат. наук, ведущий научный сотрудник, Институт проблем нефти и газа Российской Академии наук (ИПНГ РАН), г. Москва, Российская Федерация

Viktor I. Lesin, Candidate of Physics and Mathematics Sciences, Leading Researcher, Oil and Gas Research Institute of Russian Academy of Sciences, Moscow, Russian Federation.

e-mail: vilesin@inbox.ru

Лесин Сергей Викторович, младший научный сотрудник, Российский государственный университет нефти и газа им. И.М. Губкина, г. Москва, Российская Федерация

Sergey V. Lesin, Junior Researcher, National University of Oil and Gas «Gubkin University», Moscow, Russian Federation

e-mail: lesinsv@gmail.com 